\title{
A Novel Blasted and Grooved Low Profile Pedicle Screw Able to Resist High Compression Bending Loads
}

\author{
Sung-Uk Kuh", Young-Sung Kim ${ }^{1}$, Hong-June Choi ${ }^{1}$, Kyung-Hyun Kim${ }^{1}$, \\ Jeong-Yoon Park', Hyun-Yong Jeong ${ }^{2}$, Dong-Kyu Chin ${ }^{1}$, Keun-Su Kim', \\ Young-Sul Yoon', Yoon-Chul Lee ${ }^{3}$, Yong-Eun Cho ${ }^{1}$ \\ ${ }^{I}$ Department of Neurosurgery, Spine and Spinal Cord Institute, Yonsei University College of Medicine, Seoul, \\ ${ }^{2}$ Department of Mechanical Engineering, Sogang University, Seoul, \\ ${ }^{3}$ Northwestern University, Evanston, Illinois, United States of America
}

Objective: Objective: Polyaxial pedicle screws are a safe, useful adjunct to transpedicular fixation. However, the large screw head size can cause soft tissue irritation, high rod positioning, and facet joint injury. However, the mechanical resistance provided by small and low profile pedicle screws is very limited. We therefore developed a novel, low profile pedicle screw using grooving and blasting treatment that is able to resist a high compression bending load.

Methods: We evaluated the compression bending force to displacement and yield loads for seven different screw head types that differed with regard to their groove intervals and whether or not they had been blasted.

Results: The rank order of screw types that had the greatest compression bending force to displacement was as follows: (1) universal polyaxial, (2) low polyaxial with $0.1 \mathrm{~mm}$ grooves and blasting, (3) low polyaxial with blasting, (4) low polyaxial with $0.15 \mathrm{~mm}$ grooves and blasting, (5) low polyaxial with $0.05 \mathrm{~mm}$ grooves and blasting, (6) low polyaxial with $0.05 \mathrm{~mm}$ grooves, (7) and low polyaxial. Low polyaxial screws with $0.1 \mathrm{~mm}$ grooves and blasting had the maximum yield load and highest compression bending force to displacement of all seven polyaxial screw head systems evaluated.

Conclusion: Blasting and grooving treatment of pedicle screw heads resulted in screw heads with a high yield load and compression bending force relative to displacement because of increased friction. Low polyaxial pedicle screws with 0.1 $\mathrm{mm}$ grooves treated by blasting have mechanical characteristics similar to those of universal polyaxial pedicle screws.

Key Words: Bone $\cdot$ Bone screw $\cdot$ Blasting, Grooving $\cdot$ Friction

\section{INTRODUCTION}

Polyaxial pedicle screw systems allow for better angle allotment and easier multi-segmental spinal surgery than the monoaxial pedicle screws traditionally used in spinal fusion surgeries $^{5)}$. Unfortunately, polyaxial screws require a high head profile and the large screw head can cause soft tissue irritation, high positioning of the rod, and facet joint injury that may lead to adjacent segment degeneration. These problems could

- Received: May 4, 2012 - Revised: June 10, 2012

- Accepted: June 25, 2012

Corresponding Author: Sung-Uk Kuh, MD, PhD

Department of Neurosurgery, Yonsei University College of Medicine,

Spine and Spinal Cord Institute, Gangnam Severance Hospital, Seoul

135-720, Korea

Tel: +82-2-2019-3397, Fax: +82-2-3461-9221

E-mail: kuhsu@yuhs.ac potentially be overcome by the development of low profile polyaxial screws. However, the lower fixation power of low profile screw heads compared to high profile screw heads has limited the surgical application of the former. A low profile screw with the same fixation strength as universal polyaxial pedicle screws would therefore be of great medical utility.

One method to develop a low profile pedicle screw is to decrease the head size of the screw head. Although the screw head is only one small part of the entire screw structure, the screw head, after surgical insertion of the screw, is located in the extra-bony space and is in direct contact with facet-related structures ${ }^{2)}$. Furthermore, a small screw head may provide more stability than a large screw head by facilitating near-adjacent rod positioning to the spinal structure ${ }^{3)}$. However, the mechanical strength provided by a small screw head is less than that of a large screw head. Furthermore, a polyaxial pedicle screw with a small screw head may be more problematic than a monoaxial pedicle screw with a small screw head 

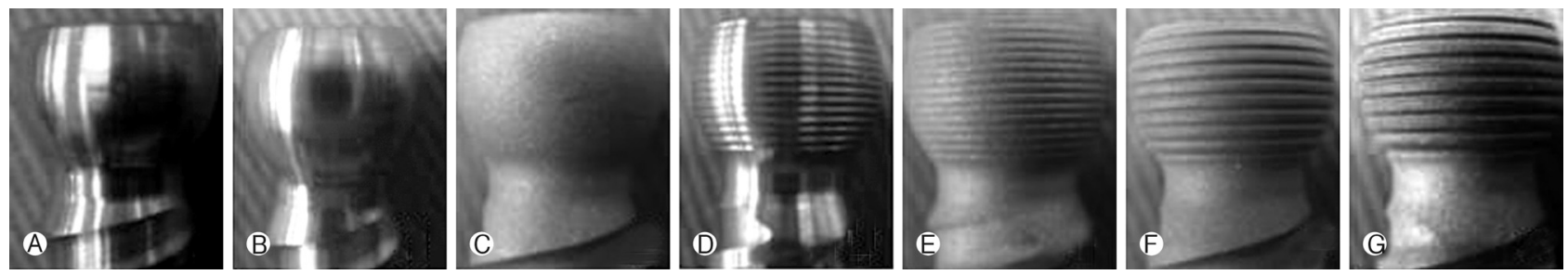

Fig. 1. The seven different types of pedicle screw evaluated: (A) universal polyaxial screw; (B) low profile polyaxial screw; (C) low profile polyaxial screw treated with blasting only; (D) low profile screw treated with grooving at $0.05 \mathrm{~mm}$ intervals; (E) low profile screw treated with blasting and grooving at $0.05 \mathrm{~mm}$ intervals; $(\mathrm{F})$ low profile screw treated with blasting and grooving at $0.10 \mathrm{~mm}$ intervals and $(G)$ low profile screw treated with blasting and grooving at $0.15 \mathrm{~mm}$ intervals.

due the ball-and-socket-like structural characteristics of polyaxial pedicle screws.

Our goal in this study was to develop a low profile polyaxial pedicle screw with a small screw head by decreasing the size of the fixture ball. Furthermore, to increase the coefficient of friction, we created grooves in the fixture ball and/or blasted it to improve the mechanical stability of the resulting low profile polyaxial pedicle screw. We evaluated the compression bending loads calculated by displacement and yield loads for a variety of screw head types that differed according to profile status, extent of grooves, and whether or not the fixture ball had been blasted.

\section{MATERIAL AND METHODS}

We evaluated seven different types of pedicle screws: universal polyaxial screws, low profile polyaxial screws, low profile polyaxial screws treated with blasting only (blasting-only screw), low profile screws treated with grooving at $0.05 \mathrm{~mm}$ intervals ( 0.05 groove-only screws), low profile screws treated with blasting and grooving at $0.05 \mathrm{~mm}$ intervals $(0.05$ groove blasting screws), low profile screws treated with blasting and grooving at $0.10 \mathrm{~mm}$ intervals ( 0.10 groove blasting screws), and low profile screws treated with blasting and grooving at $0.15 \mathrm{~mm}$ intervals ( 0.15 groove blasting screws) (Fig. 1). Screws were grooved by machining the fixture ball at regular intervals (Fig. 2) and blasting treatment was performed by blasting the fixture ball of the screw with aluminum oxide $\left(\mathrm{Al}_{2} \mathrm{O}_{3}\right)$ (Fig. 3). These screws are not commercially available; therefore, we made prototypes for the static compression test. All screws were made of titanium alloy (Ti-6AI-4V ELI). Furthermore, the length of the screw shaft was standardized to $40 \mathrm{~mm}$ and the diameter to $6 \mathrm{~mm}$. The screw shaft was potted in an ultrahigh molecular weight polyethylene (UHMWPE) block and the screw head was mounted perpendicularly on a standardized $\operatorname{rod}$ (Ti-6AI-4V ELI alloy, diameter: $5.0 \mathrm{~mm}$, length: $100.0 \mathrm{~mm}$ ) at $1,000 \mathrm{~N} \cdot \mathrm{cm}^{1)}$ (Fig. 4). Construct rigidity was measured using

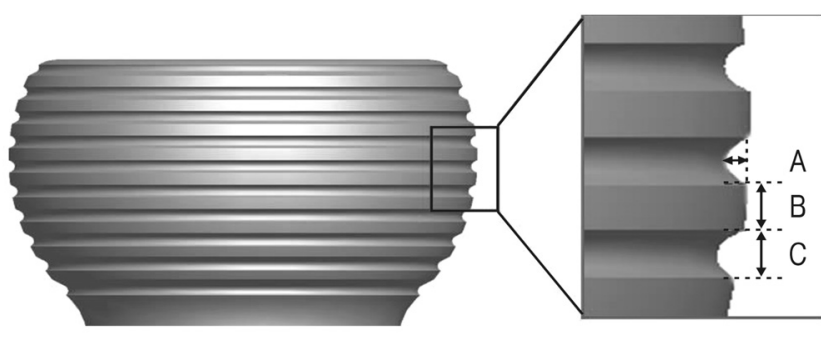

Fig. 2. Schematic illustration of the grooving method. The depth of the groove (A) was kept constant $(0.1 \mathrm{~mm})$ while the groove interval (B) was varied. The width of the groove $(C)$ was made equal to the groove interval (B).
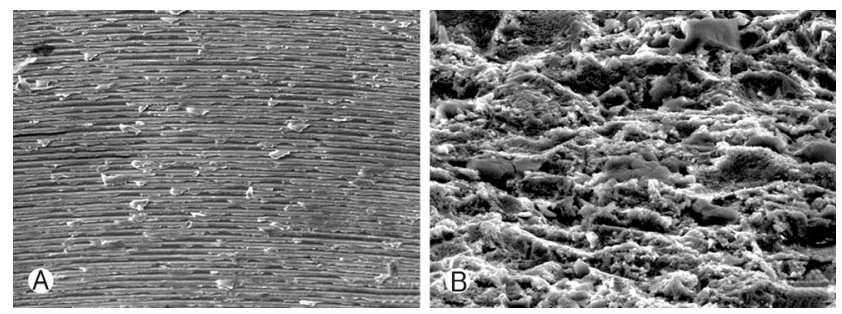

Fig. 3. Electron microscopic views of a fixture ball pre- and postblasting. The surface of the blasted fixture ball (B) was rougher than that of the non-blasted fixture ball (A).

a single column-type universal testing machine (RB $302 \mathrm{ML}^{\mathrm{TM}}$; R\&B Inc., Daejon, Korea) and ASTM F1717-09 (standard test methods for spinal implant constructs in a vertebrectomy model; ASTM international, West Conshohocken, PA, USA) was used as the test protocol. Static compression bending force was applied at a point $40 \mathrm{~mm}$ from the screw insertion point perpendicular to the long axis of the screw. The compression force was applied at a rate of $25 \mathrm{~mm} / \mathrm{min}$. Outcomes including compressive bending ultimate load and compressive bending yield load were recorded at set intervals. The compressive bending yield load was defined as the force that caused a displacement at $2 \%$ offset yield and permanent deformation ${ }^{1)}$. Each type of screw was tested five times. Comparison of yield loads based on screw type was performed using a one-way ANOVA. The level of significance was set at $\mathrm{p}<0.05$. 


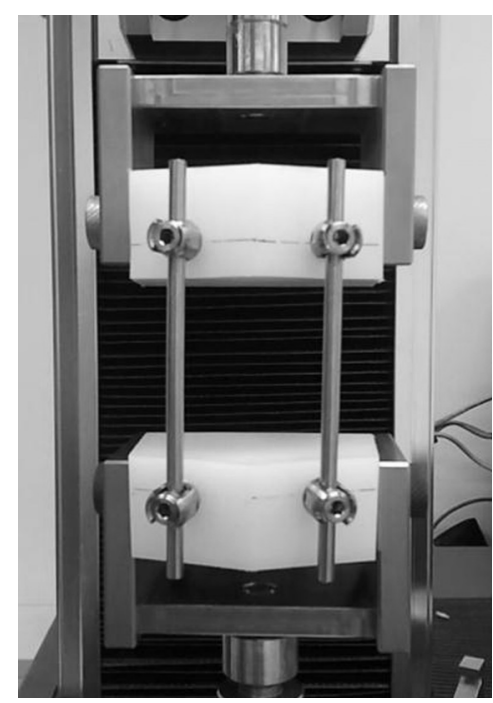

Fig. 4. Setup of the single column-type universal testing machine. The pedicle screw is encased in UHMWPE blocks, and the rod construct is attached to the screw heads. The blocks are compressed at a load rate of $25 \mathrm{~mm} / \mathrm{min}$.

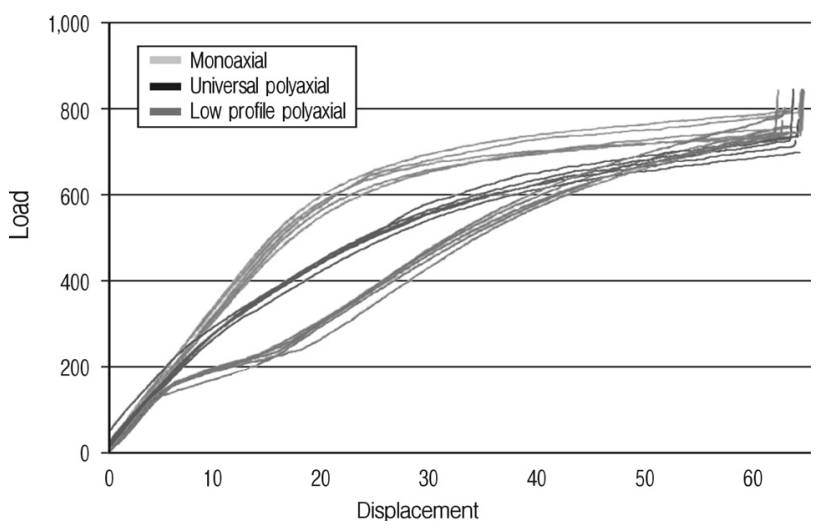

Fig. 5. Comparison of the load-displacement curves of monoaxial and polyaxial pedicle screws. The load-displacement curves show the high rigidity and durability of monoaxial pedicle screws. When the compressive bending force was approximately 200 $\mathrm{N}$, the load-displacement curves of the low profile polyaxial pedicle screws showed an early plateau caused by slip behaviors at the interface between the inner surface of the screw head and the fixture ball.

\section{RESULTS}

\section{Comparison of monoaxial and polyaxial screws}

To compare the mechanical stability of the monoaxial and polyaxial screws, we performed compressive bending tests and load displacement curves were recorded for all monoaxial,

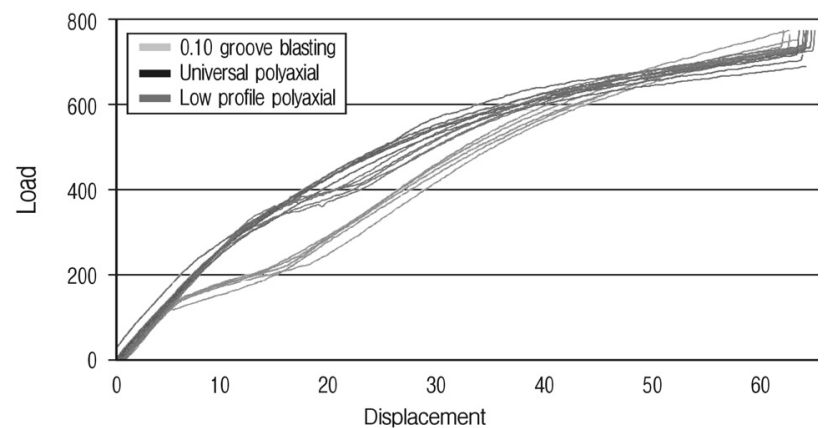

Fig. 6. Effect of $0.10 \mathrm{~mm}$ grooving and blasting treatment. The combination of blasting and $0.10 \mathrm{~mm}$ grooving was most effective at increasing the yield load of the screw head by increasing the frictional force. The load-displacement curves of $0.10 \mathrm{~mm}$ grooved and blasted screws were similar to those of universal polyaxial pedicle screws. Furthermore, the early plateau of the low profile polyaxial screws was largely overcome by the grooving and blasting treatment.

universal polyaxial, and low profile polyaxial screw types. The universal polyaxial and low profile polyaxial screws showed more displacement for the same increased load amount from 150 to $400 \mathrm{~N}$ in the load scale compared to monoaxial screws, and low profile polyaxial screws showed much more displacement than universal polyaxial screws due to their lower friction coefficient (Fig. 5). The yield loads (N) of monoaxial, universal polyaxial, and low polyaxial screws were 556 $\pm 21,339$ \pm 16 , and $164 \pm 15$, respectively. The low profile polyaxial screw showed the lowest yield load, which we attributed to a decrease in frictional force due to the small size of the fixture ball.

\section{Effects of blasting, grooving, or a combina- tion treatment}

To increase the yield load of low profile polyaxial pedicle screws, we attempted to increase the frictional force between the fixture ball and head by treating the fixture ball with blasting, grooving, or a combination of both. There was no statistical difference between the final compressive bending loads of each treatment group. Blasting-only treatment or grooving at $0.05 \mathrm{~mm}$ intervals increased the yield load significantly compared to the no-treatment group. The blasting treatment was superior to $0.05 \mathrm{~mm}$ grooving in increasing the yield load. The yield loads of screws treated with blasting and grooving at $0.05 \mathrm{~mm}$ intervals, $0.1 \mathrm{~mm}$ intervals, or $0.15 \mathrm{~mm}$ intervals was $285 \pm 11,346 \pm 20$, and $305 \pm 21$, respectively. The treatment most effective at increasing the yield load was the combination of blasting and $0.1 \mathrm{~mm}$ grooving; screws treated in this manner had a yield load similar to that of universal polyaxial screws (Figs. 6, 7). 


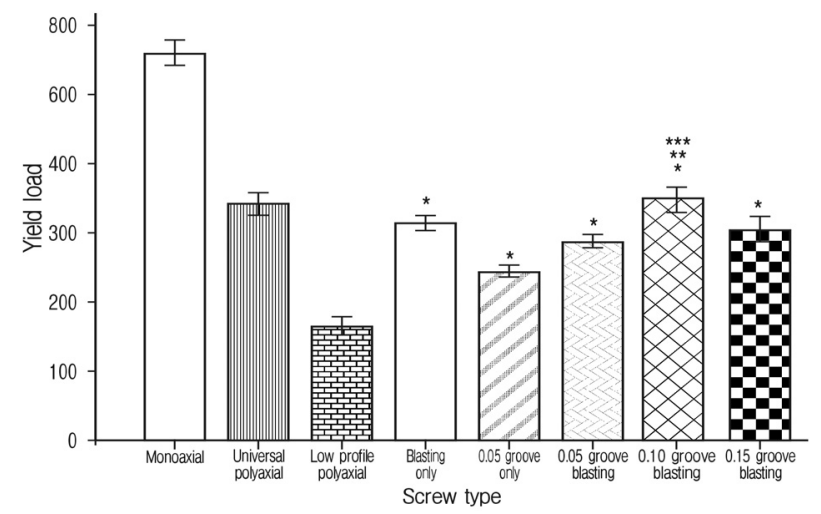

Fig. 7. Yield loads for the various types of screw heads. All treatments were effective at increasing the yield load of low profile polyaxial pedicle screws $(p<0.05)$. The treatment most effective at increasing the yield load of low profile polyaxial pedicle screws was the combination of $0.10 \mathrm{~mm}$ grooving and blasting $\left(^{* *} \mathrm{p}\right.$ $<0.05$ ). The yield load of screws treated with blasting and 0.1 $\mathrm{mm}$ grooving was not statistically different from that of universal polyaxial screws ( $p=0.99)$.

\section{DISCUSSION}

Upon testing of our prototypes, we observed permanent deformation of the screw shaft, screw head, or rod. If a compressive bending force is applied to a screw, the weakest part of the screw will be permanently deformed. In our study, when a compressive bending force was applied to monoaxial screws, no deformation of the screw head was observed; all of the deformation occurred in the rods. This result indicates that the head portion of monoaxial screws is highly rigid and durable. Although these mechanical characteristics of the monoaxial screw system result in higher stiffness, a monoaxial screw's in situ fixation strength may be less than that of polyaxial screws because of assembly difficulties ${ }^{6,7}$. For example, if a patient with severe spinal deformity undergoes pedicle screw fixation with a fixed screw system, it is difficult to assemble the screw head and rod. The surgeon may be forced to change the screw entry point or insertion depth to allow assembly during the operation. These seemingly minor changes can decrease the in situ fixation strength of the fixed screw system despite its high rigidity and durability ${ }^{6,7}$. Furthermore, if an excessive force is applied to the screw head during assembly, intra-operative screw loosening may occur and/or bony or ligamentous structures may be injured. To overcome these problems, polyaxial screw heads, which allow the screw a range of motion along several different axes, were developed by introducing the concept of joint structure to the screw head. This development has facilitated easy assembly of the screw head. However, our results indicate that the screw head of polyaxial screws is a potential weak point because of the joint structure.

When we applied a compressive bending force to the polyaxial screws, there was a sudden increase in displacement and the yield loads of these screws were lower than those of monoaxial screws. This displacement behavior was not seen in any other screw type evaluated. This phenomenon may be caused by slip behavior at the interface between the inner surface of the screw head and the fixture ball. Although some degree of frictional force may develop at the interface between the inner surface of the screw head and fixture ball when the polyaxial head is mounted on rods using adequate torque, this force is not enough to achieve the rigidity and durability of other structures in the polyaxial screw system. Thus, the first structure that develops permanent deformation under a static compressive bending load is the polyaxial head. A sudden increase in displacement in the low profile polyaxial screw system was seen at lower compressive bending loads than for universal polyaxial screws. Furthermore, the yield loads of low profile polyaxial screws were lower than those of universal polyaxial screws. We hypothesize that this result may be due to decreased frictional force resulting from the smaller screw head size of low profile polyaxial screws compared to universal polyaxial screws.

The frictional force is proportional to the friction coefficient and the force applied to an object, which is also called the normal force. Although normal force can be applied by increasing the torque when a polyaxial head is mounted on a rod, there is a certain limit to the torque because high torque itself may induce wear and tear on the screw head and rod. To increase the comparatively low frictional force of the low polyaxial profile screw head to a level equivalent to that of a universal polyaxial screw, we increased the friction coefficient between the inner surface of the screw head and fixture ball by modifying the surface of the fixture ball by blasting, grooving, or a combination of both. These treatments were effective at increasing the yield load. Among screws treated by both blasting and grooving, the screws with grooves at intervals of $0.1 \mathrm{~mm}$ had a higher yield load than the groups with 0.05 and 0.15 $\mathrm{mm}$ interval grooves. In fact, the yield load of blasted screws with $0.1 \mathrm{~mm}$ grooves was similar to that of universal polyaxial screws. This result indicates that there is an optimum interval and depth of grooving for increasing the frictional force.

It has not yet been established how much yield load the screw head should be able to bear to obtain optimal clinical results. Application of too high a yield load to the screw head will likely cause fracturing of the rod or screw shaft because the stress applied to the construct will be delivered to each component of the screw system without any loss. Although it has not been proven in clinical studies, angular deformation of the polyaxial head may be the primary buffer against the 
loading force in the case of instrument failure. In our study, all deformations occurred on the rods associated with monoaxial screws, which experience very high yield loads. In the polyaxial screw system, primary deformations occurred within the limits of the angular motion of the polyaxial screw head and rod deformations subsequently developed. Clinically, a decline in the incidence of rod and screw shaft fractures has been observed over the years since the introduction of polyaxial screws ${ }^{4)}$. This tendency is presumably due to the lower yield load of universal polyaxial screw heads compared to the actual screw or rod. However, too low a yield load can cause failure of post-operative spinal alignment, cage subsidence due to instability, and bone fusion failure. We demonstrated that grooving and blasting treatment can effectively increase the low yield load of low profile polyaxial screw heads. With technical advancements in blasting and grooving treatments, we anticipate the development of low profile polyaxial screws with ideal yield loads.

\section{CONCLUSION}

We found that treatment of the ball fixture of low profile polyaxial screws by blasting and grooving increased the yield load of the screw head by increasing the frictional force. These methods can therefore be used to produce low profile polyaxial screws with compression bending load values similar to displacement and yield load values.

\section{Acknowledgement}

This paper was presented at The Korean Neurosurgical Society Meeting in April 2011.

This work has been financially supported by Genoss Company.

\section{REFERENCES}

1. ASTM international: Standard test methods for spinal implant constructs in a vertebrectomy model. ASTM F1717-1709

2. Chen Z, Zhao J, Xu H, Liu A, Yuan J, Wang C:Technical factors related to the incidence of adjacent superior segment facet joint violation after transpedicular instrumentation in the lumbar spine. Eur Spine J 17:1476-1480, 2008

3. Crawford NR, Doüan S, Yüksel KZ, Villasana-Ramos O, SotoBarraza JC, Sawa AG, et al: In vitro biomechanical analysis of a new lumbar low-profile locking screw-plate construct versus a standard top-loading cantilevered pedicle screw-rod construct: technical report. Neurosurgery 66:404-406, 2010

4. Fogel GR, Reitman CA, Liu W, Esses SI: Physical characteristics of polyaxial-headed pedicle screws and biomechanical comparison of load with their failure. Spine 28:470-473, 2003

5. Lim HJ, Kwon SC, Roh SW, Jeon SR, Rhim SC: Outcomes of unilateral transforaminal lumbar interbody fusion in degenerative lumbar spine disease: Korean J Spine 2(1):19-25, 2005

6. Polly DW, Orchowski JR, Ellenbogen RG: Revision pedicle screws: bigger, longer shims-what is best? Spine 23:13741379, 1998

7. Shepard MF, Davies MR, Abayan A, Kabo JM, Wang JC: Effects of polyaxial pedicle screws on lumbar construct rigidity. J Spinal Disord Tech 15:233-136, 2002 